\title{
Controllable photon-pair spectral correlations
}

\author{
M. Cordier ${ }^{1}$, B. Debord ${ }^{2}$, F. Gérôme ${ }^{2}$, P. Delaye ${ }^{3}$, F. Benabid ${ }^{2}$ and I. Zaquine ${ }^{1}$ \\ 1. Laboratoire de Traitement et Communication de l'Information,Télécom ParisTech, Univ Paris-Saclay, 75013 Paris France \\ 2. GPPMM Group, XLIM Research Institute, CNRS UMR 7252, Univ Limoges, Limoges, France \\ 3. Laboratoire Charles Fabry, Institut d'Optique Graduate School, CNRS, Univ Paris-Saclay, 91127 Palaiseau France
}

Photon-pair states, whether spectrally correlated or separable, can all be very useful in quantum technology applications. For example, the former are used for improving the security of quantum key distribution, whilst the latter are the backbone in heralded single photon sources. It has been shown that the amount of spectral correlations is well-described by the shape of the Joint Spectral Amplitude function (JSA), which mostly depends on the relative group velocity relation between the pump, signal and idler photons within the source medium [1].

Here, we report on a photon-pair source whose states can be controlled from separable to spectrally entangled. The source is based on four-wave mixing nonlinear effect within a gas-filled hollow-core photonic crystal fiber (HCPCF). It combines three important properties: (1) Raman-free generation thanks to the use of a noble gas and to a minute overlap with silica within the hollow-core [2]; (2) strong efficiency nonlinear medium and (3) a high versatility in the phase-matching conditions thanks to the fiber microstructuration and gas pressure tunability.

The inhibited-coupling HCPCF (see Fig. 1a) filled with xenon, was designed to operate at wavelengths that are convenient for heralded single photon sources; the idler lies in the telecom wavelength range $(\sim 1545 \mathrm{~nm})$, while the signal wavelength is in the range of atomic transitions and Silicon single photon detectors $(\sim 778 \mathrm{~nm})$.

More importantly, we show how the multiband dispersion profile (see Fig. 1b) of such medium allows to tailor phase- and group velocity relations and possibly at any given wavelength from the UV to infrared [3]. We demonstrate experimentally an active control over the generated photon spectral-correlation that allows spectrally entangled and factorable states to be obtained within the same device (examples in Fig. 1c). More specifically, a gallery of different JSI, including exotic shape, is measured by tuning various parameters: gas pressure, pump spectral FWHM, spectral chirp and pump spectral envelope. Such a versatile photon-pair source can target both applications requiring factorable (heralded single photon) and correlated states (spectral entanglement) and paves the way to spectro-temporal mode encoding [4]. Furthermore, the photon-pair state is generated over an unprecedented tunable frequency-range that span well over tens of $\mathrm{THz}$. We will present complete theoretical and experimental results demonstrating the full capacity of this platform to generate photon pair with controllable spectral properties.
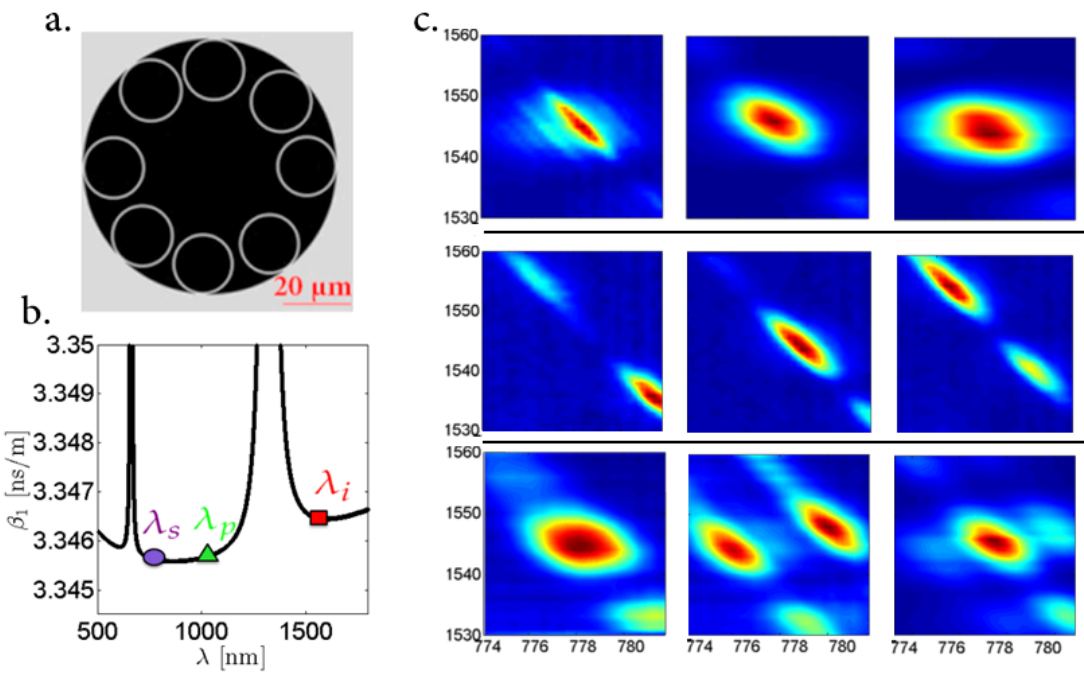

pump spectral

$$
\text { width }
$$
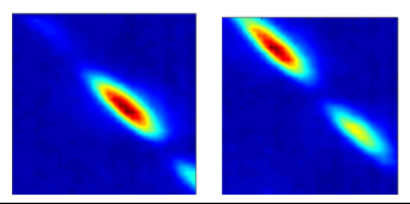

gas

pressure

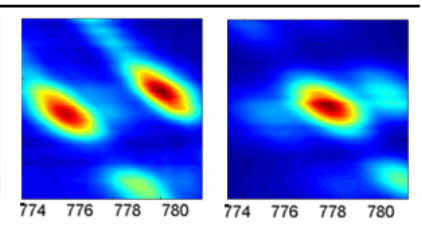

pump

envelope

Fig. 1 a. Optical image of the fiber cross-section. b. Simulated inverse-group velocity exhibiting a multiband profile. c. Measured JSI of the source for different experimental parameters; The JSI are obtained using the stimulated emission tomography technique.

\section{References}

[1] W. P. Grice et al., "Eliminating frequency and space-time correlations in multiphoton states," PRA 64, 063815 (2001)

[2] B. Debord et al., "Ultralow transmission loss in inhibited-coupling guiding hollow fibers. " Optica 4.2 (2017): 209-217.

[3] M. Cordier et al., "Active engineering of four-wave mixing spectral entanglement", arXiv:1807.11402, (2018).

[4] C. Silberhorn et al., "Photon temporal modes: a complete framework for quantum information science, " PRX 5(4), 2015 